\title{
Editorial on the Special Issue Innovations of Evolving-Adaptive Systems in the Era of the 4th Industrial Revolution
}

\author{
Lazaros Iliadis ${ }^{1}$
}

Published online: 22 August 2021

๑) The Author(s), under exclusive licence to Springer-Verlag GmbH Germany, part of Springer Nature 2021

We are living in the era of the 4th industrial revolution, which is characterized by the advent of cyber-physical systems. Machine Learning algorithms have already been embedded in our post-modern societies and they have altered the way people live, work, travel and interact. Several Ethical problems have emerged calling for serious attention. Evolving Systems are characterized by a cooperative and adaptive nature, and they are employing a wide spectrum of Intelligent recursive algorithms. They have a significant impact to the development of the technological "tsunami" we are experiencing.

This is the Editorial of the Innovations of EvolvingAdaptive Systems in the Era of the 4th Industrial Revolution Special Issue, of the EVOS Journal. Totally nine (9) high quality papers, on timely and novel evolving intelligent modeling approaches have been accepted for publication, whereas several other submissions have been rejected after passing a peer review process.

The first paper is entitled "Eyelid Basal Cell Carcinoma classification using Shallow and Deep Learning Artificial Neural Networks". It is authored by Adam Adamopoulos, Evaggelos Chatzopoulos, George Anastasopoulos from the Democritus University of Thrace Greece, and by Efstathios Detorakis from the University of Crete Greece. It discusses the development and application of a Machine Learning Model and a respective System, that successfully performs classification of Eyelid Basal Cell Carcinoma cases. It is an innovative medical modeling research based on quite strong foundations.

Mohamed Hazgui and Haythem Ghazouani, from the Universite de Tunis El Manar, Tunisia and Walid Barhoumi,

Lazaros Iliadis

liliadis@civil.duth.gr

1 Lab of Mathematics and Informatics, Department of Civil Engineering, School of Engineering, Democritus University of Thrace, University Campus, Kimmeria, 67100 Xanthi, Greece from the Universite de Tunis El Manar and the Universite de Carthage, Tunisia, are the authors of the second paper entitled "Evolutionary-based Generation of Rotation and Scale Invariant Texture Descriptors from SIFT Keypoints". The paper introduces a genetic programming algorithm, with the intention of evolving a rotation and scale-invariant set of image descriptors. The generated vectors are used for classifying texture images precisely using a limited number of instances. The algorithm was tested successfully on five datasets.

The third paper is authored by Maria Kotouza, Alexandros-Charalampos Kyprianidis, Sotirios-Filippos Tsarouchis, Antonios C. Chrysopoulos and Pericles A. Mitkas, from the department of Electrical and Computer Engineering of the Aristotle University of Thessaloniki, Greece. It is entitled "Science4Fashion: An End-to-End Decision Support System for Fashion Designers". The paper introduces the Science4Fashion end-to-end AI system. It facilitates fashion designers by collecting and analyzing data from many different sources and by suggesting products according to their needs. An overview of the system's modules is presented, emphasizing data collection, data annotation using deep learning models, and product recommendation and user feedback processes.

Georgios Barlas and Efstathios Stamatatos from the University of Aegean, Greece, have authored the fourth paper whose title is: "A Transfer Learning Approach to Crossdomain Authorship Attribution". The contribution of the paper is the following: The authors introduce a Transfer Learning model which employs pre-trained neural networks and a multi class classifier. The proposed model successfully performs authorship attribution, by identifying authors of texts. There is a series of potential application areas, e.g. forensics, cyber security and social media analytics.

The title of the fifth paper is "Biofeedback: $e$-health prediction based on evolving fuzzy neural network and wearable technologies". The authors are Mario Malcangi and Giovanni Nano, from the University of Milan, Italy. This 
paper describes the development of an Intelligent model and a respective system, that exploits recent advances in wearable microelectronics and Evolving Fuzzy Neural networks, capable to learn online. The system is measuring the vital signs such as the heart rate, the bio impedance, body temperature, motion activity, blood pressure, blood oxygenation and respiration rate. Simulation results demonstrate that biofeedback control model could be an effective reference design that enables short and long-term e-health prediction.

The sixth paper is authored by John Oomen and Ekaba Bisong from University of Ottawa, Canada. Its title is "On Utilizing the Transitivity Pursuit-Enhanced Object Partitioning to Optimize Self-Organizing Lists-on-Lists". The authors optimize the singly linked-list data structure to mitigate the worst-case linear cost of list retrieval as well as to lower the average-case asymptotic cost. The goal of the rearrangement is to move elements that are more frequently accessed towards the head of the list.

The seventh paper is entitled "Public Key Authenticated Encryption with Multiple Keywords Search using Mamdani System". The authors are Yang Ma and Hassan Kazemian from London Metropolitan University, United Kingdom. This paper extends the Public Key Encryption with Multi Keywords Search approach, using a Mamdani system (known as the $m$-PEMKS) scheme. It proposes a robust $m$-PEMKS mechanism which has the properties of Ciphertext and Trapdoor Indistinguishability. It employs user Authentication which can prevent Off-line Keyword Guessing Attack and Inside Keyword Guessing Attack. Besides, the proposed scheme supports both Fuzzy Keyword Search and Multiple Keywords Search and therefore, it is more practical and could be applied to the general public networks.

The eighth paper is authored by Mike Spiliotis from the Democritus University of Thrace Greece, and by Luis Garrote from the Technical University of Madrid, Spain. Its title is "Unit Hydrograph Identification based on Fuzzy
Regression Analysis". The introduced approach aims to treat the uncertainties of the unit hydrograph rainfall- runoff transformation, based on the fuzzy linear regression model. The components of the unit hydrograph are proposed to be symmetric triangular fuzzy numbers. The main idea is to develop a fuzzified version of the widely-used unit hydrograph. The model was successfully tested based on twenty real rainfall-runoff events.

The ninth paper is authored by Nikos Mylonas and Basil Papadopoulos, from the Democritus University of Thrace, Greece. It is entitled "Fuzzy hypotheses tests for crisp data using non-asymptotic fuzzy estimators, fuzzy critical values and a degree of rejection or acceptance". The fuzzy hypotheses testing is based on critical values and fuzzy test statistics, produced by fuzzy estimators. This approach is particularly useful in critical situations, where subtle comparisons between almost equal statistical quantities have to be made. The introduced model employs non-asymptotic fuzzy estimators which are triangular shaped fuzzy numbers, that generalize the fuzzy estimators based on confidence intervals in such a way that they are functions.

I wish to express my warm gratitude to the Editors in Chief of the Springer Evolving systems Journal, distinguished Professors Plamen Angelov, Dimitar Filev, and Nikola K. Kasabov, for offering me the chance to edit this very interesting Special Issue. I hope that its contribution to the literature will be significant and that it will offer inspiration to several researchers globally.

Publisher's Note Springer Nature remains neutral with regard to jurisdictional claims in published maps and institutional affiliations. 